Astin Bulletin $11(1980)$ 1-16

\title{
A GAME THEORETIC LOOK AT LIFE INSURANCE UNDERWRITING*
}

\author{
JEAN LEMAIRE \\ Université Libre de Bruxelles
}

\begin{abstract}
The decision problem of acceptance or rejection of life insurance proposals is formulated as a two-person non cooperative game between the insurer and the set of the proposers. Using the minimax criterion or the Bayes criterion, it is shown how the value and the optimal strategies can be computed, and how an optimal set of medical informations can be selected and utilized.
\end{abstract}

\section{FORMULATION OF THE GAME}

The purpose of this paper, whose mathematical level is elementary, is to demonstrate how game theory can help the insurers to formulate and solve some of their underwriting problems. The framework adopted here is life insurance acceptance, but the concepts developed could be applied to any other branch.

The decision problem of acceptance or rejection of life insurance proposals can be formulated as a two-person non cooperative game the following way: player $1, P_{1}$, is the insurer, while player $2, P_{2}$, is the set of all the potential policy-holders. The game is played many times, in fact each time a member of $P_{2}$ fills in a proposal. We suppose that this person is either perfectly healthy (and should be accepted) or affected by a disease which should be detected and cause rejection. We shall assume for the moment that the players possess only two strategies each: acceptance and rejection for $P_{1}$, health or disease for $P_{2}$. To be more realistic we should introduce a third pure strategy for $P_{1}$ : acceptance of the proposer with a surcharge. To keep the analysis as simple as possible we shall delay the introduction of surcharges until section 4 . Consequently we can define a $2 \times 2$ payoff matrix for the insurer.

\begin{tabular}{lcc}
\hline$P_{1} \backslash P_{2}$ & $\begin{array}{c}\text { healthy } \\
\text { proposer }\end{array}$ & $\begin{array}{c}\text { ill } \\
\text { proposer }\end{array}$ \\
\hline acceptance & $A$ & $C$ \\
rejection & $B$ & $D$ \\
\hline
\end{tabular}

It is evident that the worst outcome for the insurer is to accept a bad risk. Interpreting the payoffs as utilities for $P_{1}, C$ should be the lowest figure. Clearly $D>B$ : it is better for the insurer to reject a bad risk than a good risk. Also $A$ must be greater than $B$. One might argue about the relative

* Presented at the 14th ASTIN Colloquium, Taormina, October 1978. 
values, $A$ and $D$, of the good outcomes. We shall suppose in the examples and the figures that $D>A$, but the analysis does not rely on this assumption.

In order to find the value of the game and the optimal strategy for $P_{1}$, we can apply

- the minimax criterion, or

- the Bayes criterion.

\section{THE MINIMAX CRITERION}

To apply the minimax criterion assimilates $P_{2}$ to a malevolent opponent whose unique goal is to deceive the insurer and to reduce his payoff. This is of course an extremely conservative approach, to be used by a pessimistic insurer, concerned only by its security level.

\subsection{Value and Optimal Strategies without Information}

Since $P_{2}$ 's objective is to harm $P_{1}$, the game becomes a $2 \times 2$ zero-sum twoperson game, which can be represented graphically. The vertical axis of fig. 1 is the payoff to $P_{1}$. His possible choices are represented by the two straight lines. The horizontal axis is $P_{2}$ 's choice: he can always present an healthy proposer, or a non healthy, or pick any probability mix in between. The use of mixed strategies is fully justified here since the game is to be played many times. Since $P_{2}$ 's payoff is the negative of $P_{1}$ 's', his objective is to minimize the insurer's maximum gain, the heavy broken line. The ordinate of point $M$

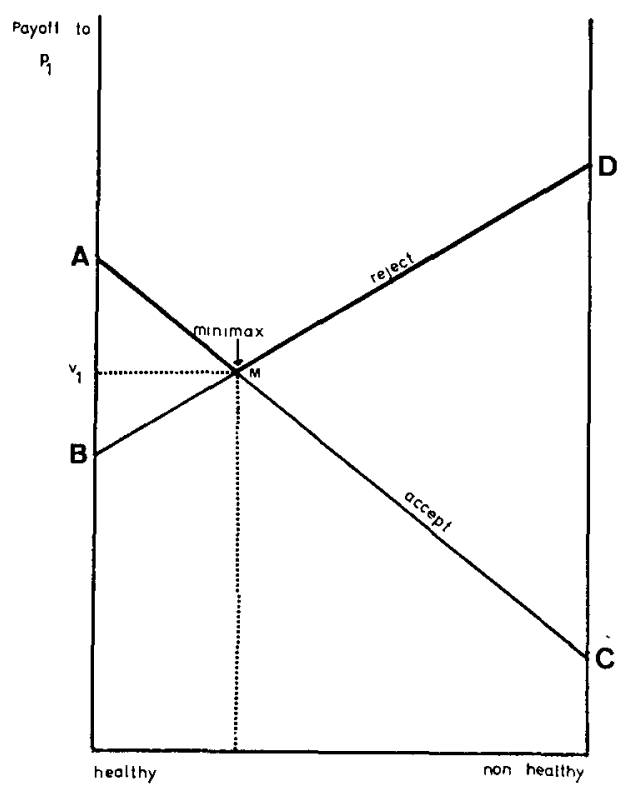

Fig. 1 
is then the value of the game. The abscissa of $M$ provides the optimal mixed strategy of $P_{2}$. $P_{1}$ 's optimal strategy can be obtained similarly (for more details see for instance OWEN (1968, p. 29)).

Thus, by adopting a mixed strategy (to accept any risk with a probability $p_{A}=\frac{D-B}{A+D-B-C}$, and to reject with a probability $\left.p_{R}=1-p_{A}\right), P_{1}$ can guarantee himself a payoff of $v_{1}=\frac{A D-B C}{A+D-B-C}$, whatever the strategy adopted by his opponent. $P_{2}$ 's optimal strategy is to present a proportion $p_{H}=\frac{D-C}{A+D-B-C}$ of good risks.

\subsection{Introduction of Medical Information}

The preceding model is extremely naive (and will only be used as reference for comparisons) since it does not take into account $P_{1}$ 's possibility to gather some information about the proposer's health, by asking him to fill in an health questionnaire, or by requiring him to undertake a medical examination. This information is of course only partially reliable. But, however imperfect, it can be used to improve $P_{1}$ 's guaranteed payoff. How can the insurer make optimal use of the information he does have? It is sufficient for our purposes to characterize the medical information by two parameters: $p_{S}$, the probability of successfully noticing a bad risk, and $p_{F}$, the false alarm probability of detecting a non-existant illness. Let us introduce a third pure strategy for $P_{1}$ : to follow the indications of the medical information. If the proposer is not healthy, his illness is detected with a probability $p_{S}$, and remains undetected with a probability $1-p_{S}$. $P_{1}$ 's expected payoff thus equals

$$
E=D p_{S}+C\left(1-p_{S}\right) \text {. }
$$

Similarly, his payoff in case the proposer is healthy is

$$
F=\left(1-p_{F}\right) A+p_{F} B
$$

Fig. 2 represents a "detector" with a .7 success probability and a .4 false alarm probability.

We notice that, in this case, $P_{1}$ can guarantee himself a payoff $v_{2}>v_{1}$ by mixing the strategies "to accept" and "to follow the detector's indication". Of course, for other values of $p_{S}$ and $p_{F}$, the optimal mixed strategy varies and can mix a different set of pure strategies. The detector can even be so imperfect that the line $F E$ passes below the intersection of $B D$ and $A C$; then the medical information is so weak that it is useless. 


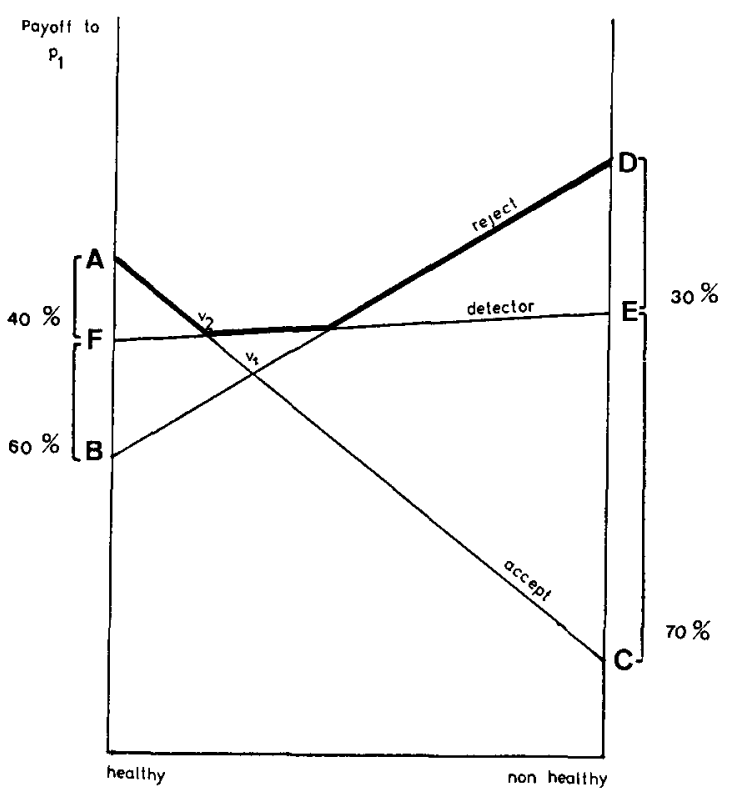

Fig. 2

\subsection{Optimal Detection System}

A detector is characterized by a pair $\left(p_{S}, p_{F}\right)$ of probabilities. The underwriters can decide to render the standards of acceptation more severe, by rejecting more people, thereby increasing the success probability $p_{S}$. Unfortunately, the false alarm probability $p_{F}$ will then increase too. Can game theory help us to select an optimal detection system? Must the company choose a "nervous" detector, with a high success probability, but also a high false alarm rate, or a "phlegmatic" or "slow" system with low probabilities $p_{S}$ and $p_{F}$ ?

Let us assume for simplicity that all the medical information has been aggregated into a single discriminating variable (for instance by using discriminant- or regression analysis). The distribution of the discriminating variable for the healthy population will usually overlap the distribution for the non healthy group. The choice of a particular detector can consist of selecting a critical value, any higher observed value leading to rejection, any lower value to acceptance (this procedure is optimal if the distributions are normal with equal variances. Otherwise, the decision rule can be obtained by a likelihood ratio method (see appendix or LEE (1971, pp. 201-203)).

The shaded zone represents the false alarm probability, the dotted region the success probability. Each critical value determines those two probabilities. If the critical value is moved to the right, the detector becomes slower. If it is moved to the left, it becomes more nervous. The set of all the critical values 


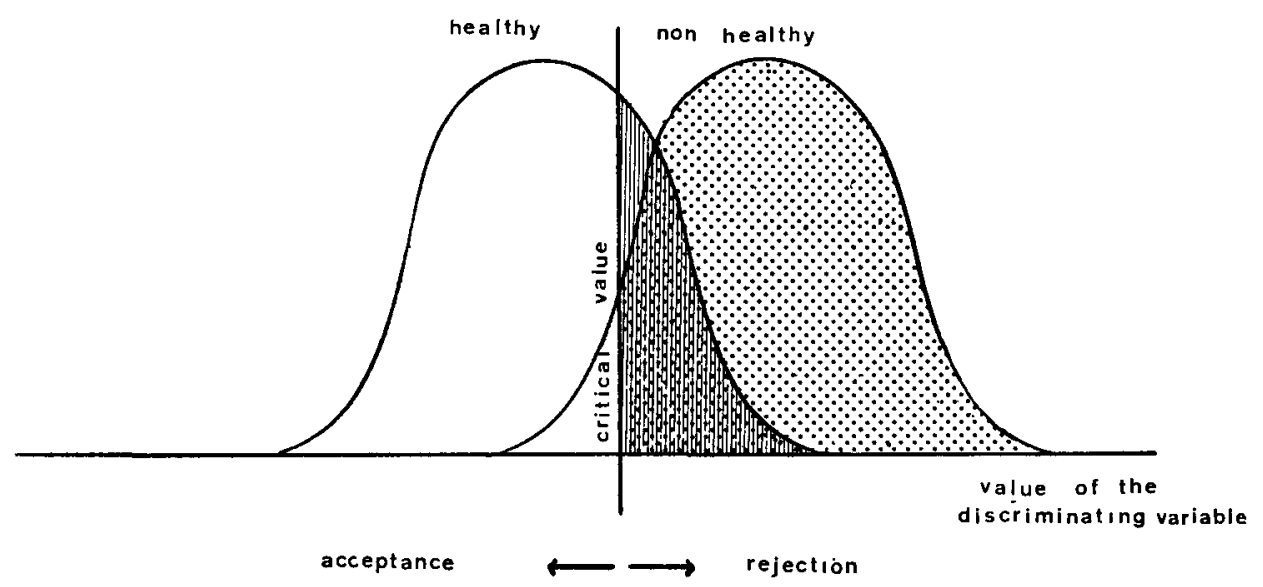

Fig. 3

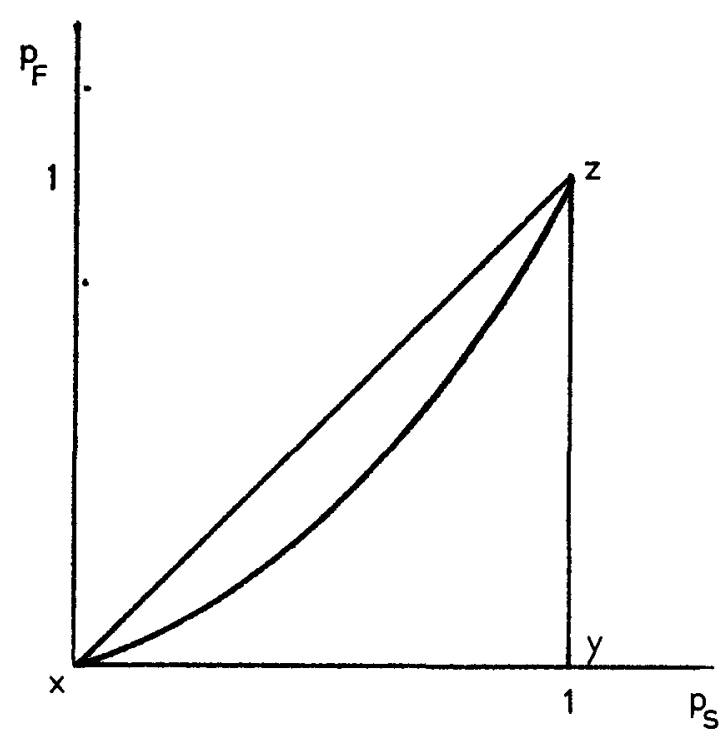

Fig. 4

defines the efficiency curve of the discriminant variable. The weaker the discriminant power of this variable, the nearest to the bissecting line its efficiency line. A perfect discriminant variable has a triangular efficiency $x y z$.

The set of all the detectors determines a set of values for the game. The highest value $v^{*}$ for the insurer is reached when the payoff line is horizontal. This can be roughly seen as follows (for a more rigorous proof see LUCE and RAIFFA (1957, pp. 394-396)): the critical value, moving from left to right, generates a family of lines with decreasing slope. If $P_{\mathbf{1}}$ chooses a detector with 
a positive slope, $P_{2}$ can reduce his payoff below $v^{*}$ by always presenting healthy proposers. Similarly, if the slope is negative, a continuous flow of non healthy proposers will keep $P_{1}$ 's payoff below $v^{*}$.

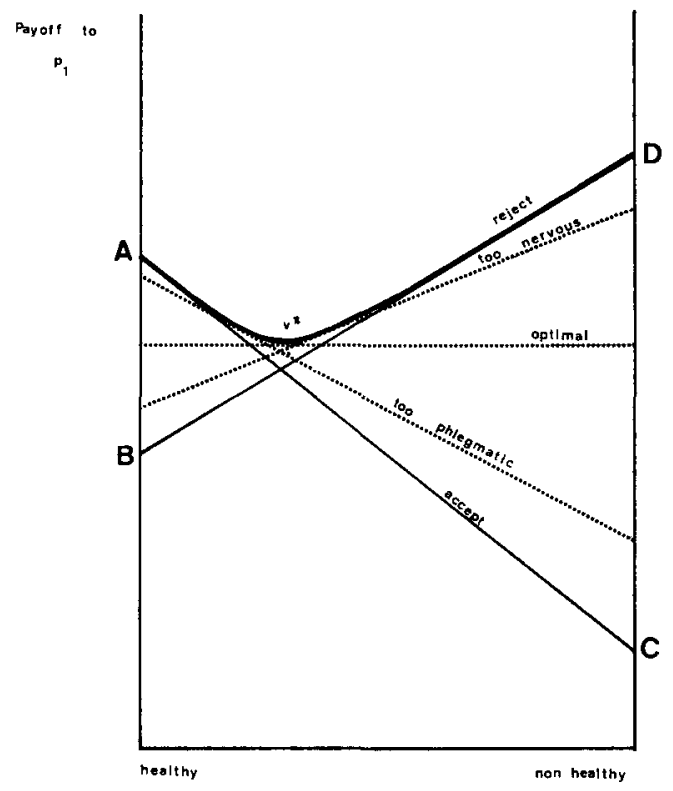

Fig. 5

The optimal detector can be easily obtained by equating the payoffs $E$ and $F$ :

$$
D p_{S}+C\left(1-p_{S}\right)=A\left(1-p_{F}\right)+B p_{F}
$$

Then

$$
p_{F}=\frac{D-C}{B-A} p_{S}+\frac{C-A}{B-A}
$$

defines a straight line in fig. 4 , whose intersection with the efficiency line determines the optimum.

Note that the optimal strategy of $P_{1}$ is a pure strategy: to follow the advice of the detector; the insurer does not have to throw a coin after the mecidal examination in order to decide if the proposer is accepted. What happens is that the "noise" in the observation system, however small, provides the necessary randomization in order to prevent $P_{2}$ from outguessing the insurer.

\subsection{The Value of Improving the Detection System}

A medical examination can always be improved: one can introduce an electrocardiogram, a blood test, ... for each proposer. But is it worth the cost? An improved discrimination ability means that the distributions of fig. 3 are more 


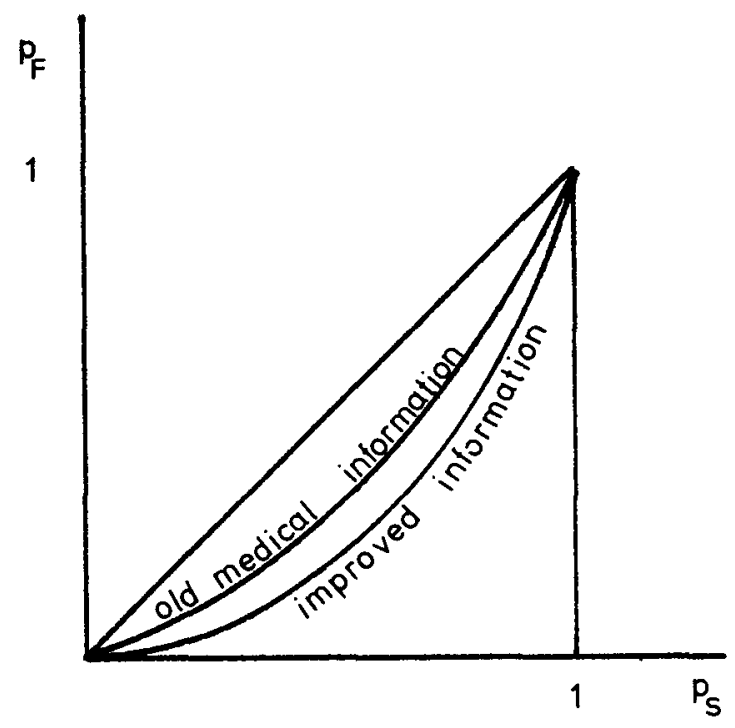

Fig. 6

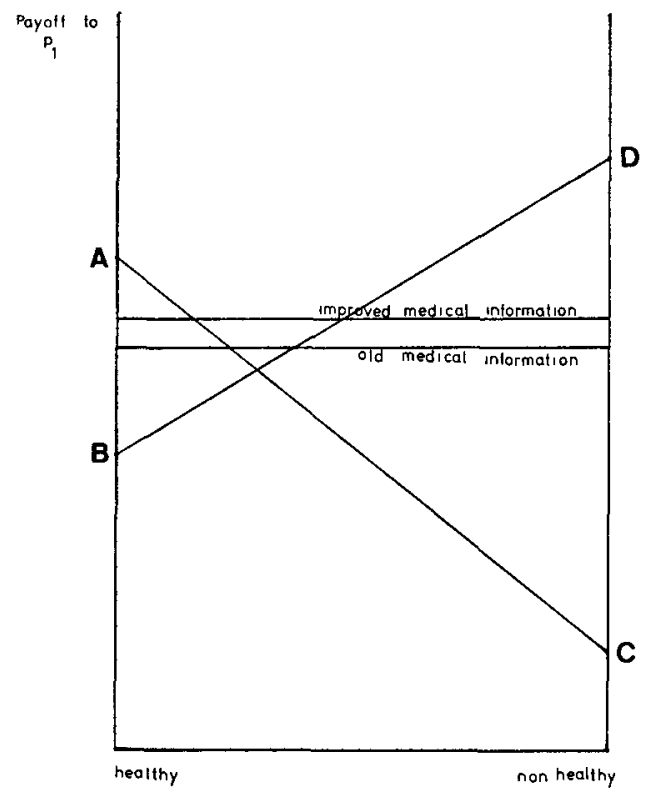

Fig. 7

separated and present less overlap. The characterizing probabilities $p_{S}$ and $p_{F}$ are improved, and the efficiency line moves away from the bisecting line.

The intersection of the improved efficiency line with (1) (which is determined only by the payoffs and therefore does not change with increased discrimina- 
tion) provides the new optimal detector; the associated value is higher for the insurer. If the cost of implementing the new system is less (in utilities) than the difference between the two values, it is worthwhile to introduce it. The insurer should be willing to pay any amount inferior to the difference of the values for the increase in his discrimination ability.

\subsection{An Example ${ }^{1}$}

All the proposers above 55 years of age willing to sign a contract of over 3 million Belgian Francs in a given company have to pass a complete medical examination with electrocardiogram. We have selected 200 male proposers, 100 rejected because of the electrocardiogram, and 100 accepted. This focuses the attention on one category of rejection causes: the heart diseases, and implicitly supposes that the electrocardiogram is a perfect discriminator. This (not unrealistic) hypothesis being made, we can consider the rejected persons to be non healthy. Correspondingly the accepted proposers will form the healthy group. We have then noted the following characteristics of each proposer:

$x_{1}$ : overweight or underweight (number of kilograms minus number of centimeters minus 100);

$x_{2}$ : number of cigarettes (average daily number);

$x_{3}$ : the presence of sugar

$x_{4}$ : or albumine in the urine;

$x_{5}$ : the familial antecedents, for the mother,

$x_{6}$ : and the father of the proposer.

We then define a variable

$$
x_{0}=\left\{\begin{array}{l}
0 \text { if the proposer is healthy } \\
1 \text { otherwise }
\end{array}\right.
$$

and apply a standard selection technique of discriminant analysis in order to sort out the variables that significantly affect $x_{0}$. The procedure only retains three variables $x_{1}, x_{2}$ and $x_{6}$, and combines them linearly into a discriminating variable. The value of this variable is computed for all the observations, and the observed distributions are presented in fig. 8. As was expected, the discrimination is quite poor, the distributions strongly overlap. The multiple correlation between $x_{0}$ and the set of the explaining variables equals .26. The group centroids are respectively .4657 and .5343 .

We then estimate for each possible critical value $p_{S}$ and $p_{F}$ and plot them on fig. 10.

1 This example presents very weak detectors and is only introduced in order to illustrate the preceding theory. 


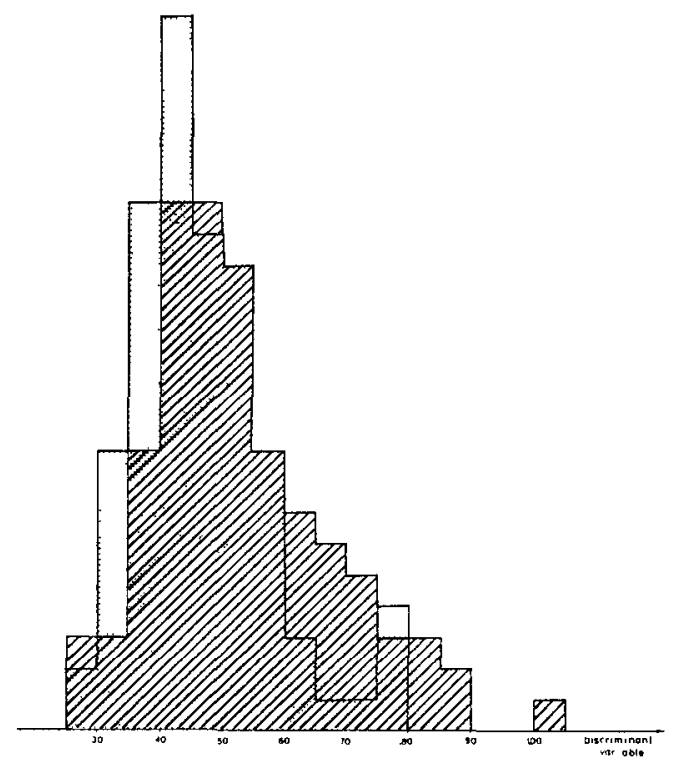

Fig. 8

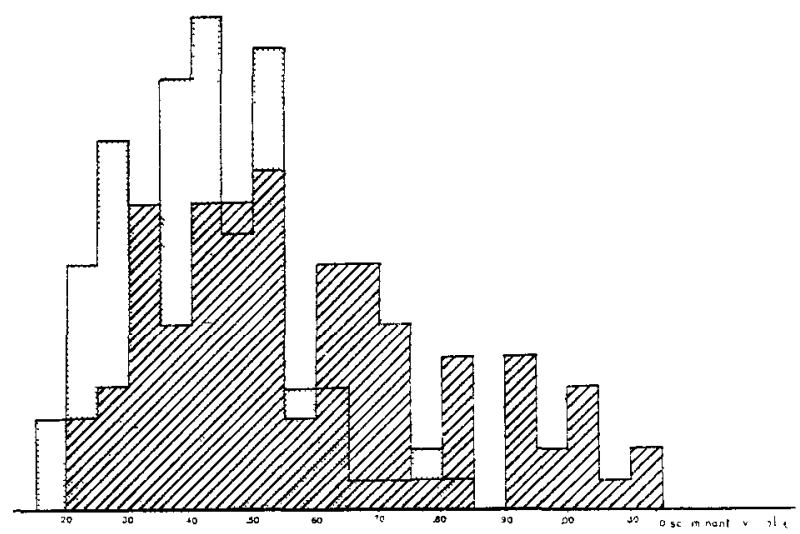

Fig. 9

We must now assign utilities to the various outcomes. We shall select $A=8, B=4, C=0$ and $D=10$. Then the value of the game without medical information is 5.714, $P_{2}$ presenting $2 / 7$ of bad 1 isks and $P_{1}$ accepting $3 / 7$ of the proposals.

Let us now introduce the medical information and for instance evaluate the strategy that corresponds to a .5 critical value. On fig. 10 , we can read $p_{S}=.51$ and $p_{F}=.33$. Then $E=.51 \times 10+.49 \times 0=5.1$, and $F=.33 \times 4+$ $.67 \times 8=6.68$. The value of this game is $6.121, P_{2}$ presenting more bad risks $(34.1 \%), P_{1}$ mixing the strategies "reject" and "follow detector" with respect- 


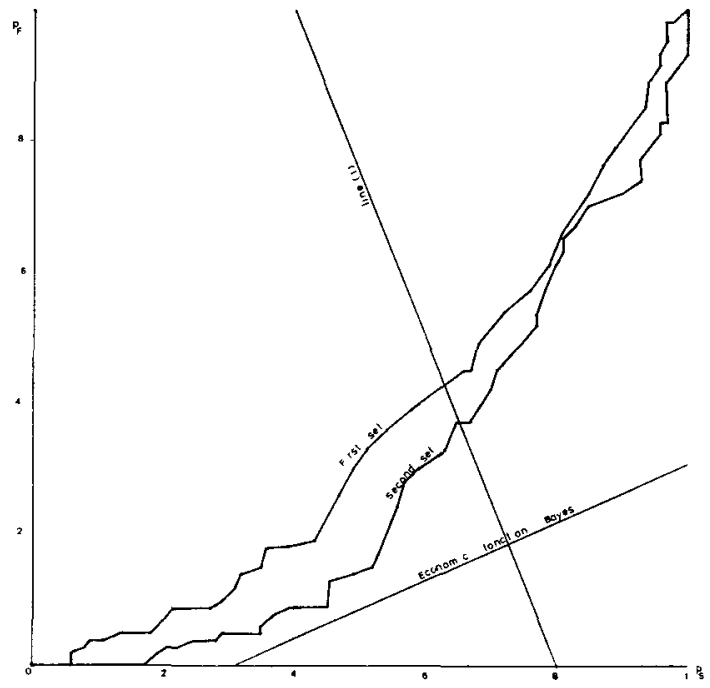

Fig. 10

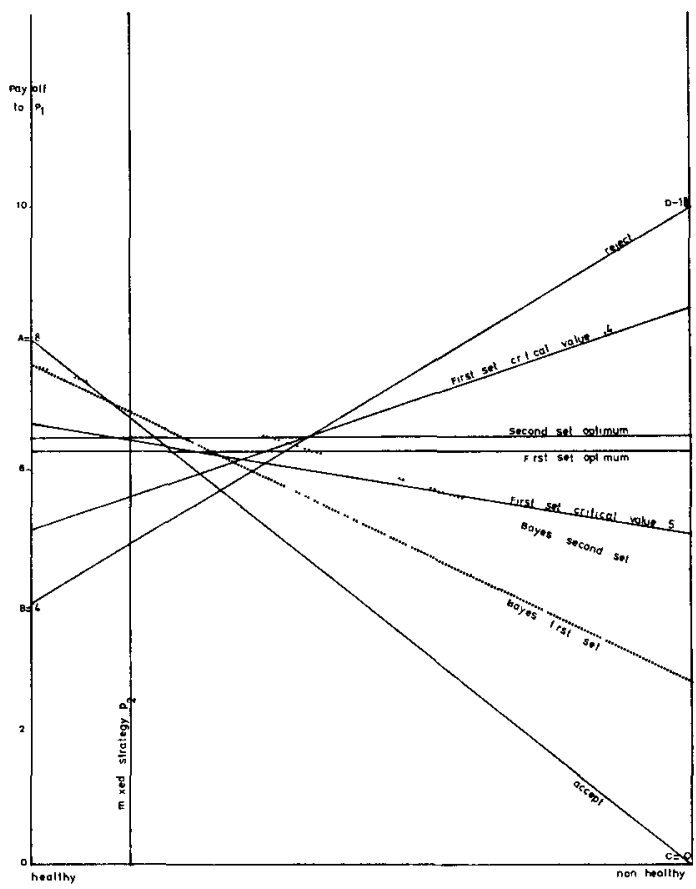

Fig. 11 
ive probabilities .208 and .792. Fig. 11 shows that this strategy is too "slow", that too many risks are accepted.

On the other hand, a detector with a .4 critical value is too nervous: too many risks are rejected. The value is $5.975 ; P_{2}$ 's optimal strategy is to present $74.7 \%$ of good risks, while $P_{1}$ should accept $29.7 \%$ of the time and trust the detector otherwise.

To find the optimum, we read the intersection of the efficiency line with equation (1), in this case

$$
p_{F}=2-\frac{5}{2} p_{S}
$$

We find

$$
\begin{aligned}
& p_{F}=.425 \\
& p_{S}=.63
\end{aligned}
$$

with a critical value of .475 . Then

$$
E=10 \times .63+0 \times .37=.425 \times 4+.575 \times 8=6.3 \text { : }
$$

if the insurer adopts the pure strategy of always accepting the advice of the medical information, he can guarantee himself a value of 6.3 irrespective of his opponent's strategy.

Let us now attempt to improve the medical examination by adding a new variable $x_{7}$, the blood pressure of the proposer. Because of the high positive correlation between $x_{1}$ and $x_{7}$, the selection procedure only retains as significant the variables $x_{2}, x_{6}$ and $x_{7}$. Fig. 9 shows that the distributions are more separated. In fact, the group centroids are now .4172 and .5828 and the multiple correlation between $x_{0}$ and the selected variables rises to .407. The efficiency line (fig. 10) is uniformly to the right of the former one. The intersection with (1) is

$$
\begin{aligned}
& p_{F}=.37 \\
& p_{S}=.652
\end{aligned}
$$

with a critical value of approximatively .45 . The value of the game rises to 6.52 , an improvement of .22 for the insurer at the cost of controlling the blood pressure of each proposer (see fig. 11).

\section{THE BAYES CRITERION}

Instead of playing as if the proposer's sole objective were to outsmart him, the insurer can apply the Bayes criterion, i.e. assume that $P_{2}$ has adopted a fixed a priori strategy. He can suppose (from past experience or from the results of a sample survey performed with a maximal medical examination) that a proportion $p_{H}$ of the proposers is healthy. The analysis is easier in this 
case, since $P_{2}$ 's mixed strategy is now assumed to be known. $P_{1}$ only faces a one-dimensional problem: he must maximize his utility on the dotted vertical line of fig. 12.

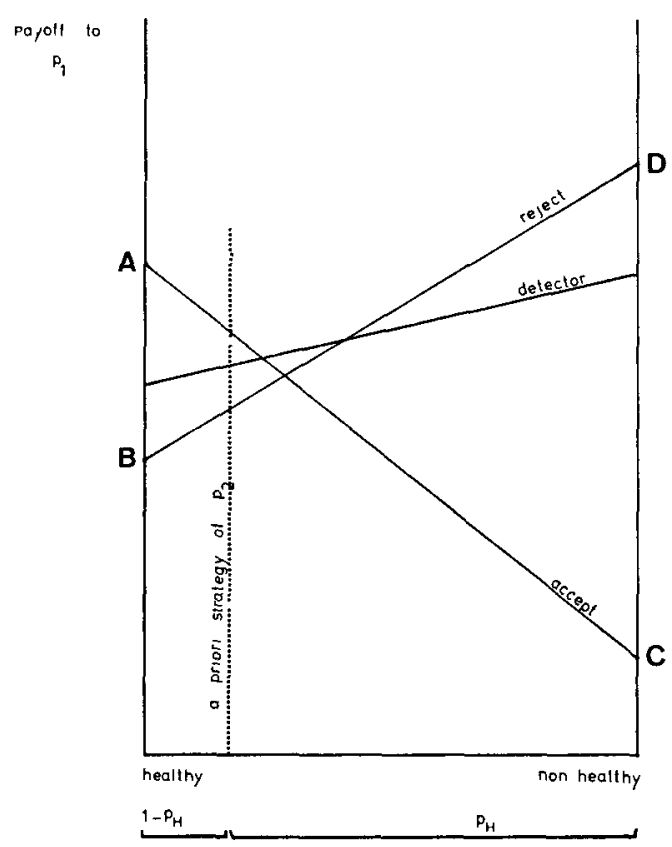

Fig. 12

One notices from fig. 12 that a medical examination is sometimes useless, especially if $p_{H}$ is near 1 . In this case, $P_{1}$ 's optimal strategy is to accept all the proposers. In the general case, $P_{1}$ should maximize the linear function of $p_{F}$ and $p_{H}$

$$
\left[p_{F} B+\left(1-p_{F}\right) A\right] p_{H}+\left[p_{S} D+\left(1-p_{S}\right) C\right]\left(1-p_{H}\right),
$$

under the condition that $p_{F}$ and $p_{S}$ are linked by the efficiency curve of fig. 4 .

As far as the example is concerned, this economic function (represented in fig. 10) becomes

$$
6.8+1.5 p_{S}-3.4 p_{F}
$$

if one supposes that $p_{2}$ 's mixed strategy is to present $15 \%$ of bad risks.

For the first set of medical information $\left(x_{1}, x_{2}, x_{6}\right)$, the maximum is reached at the point $p_{S}=.28, p_{F}=.09$. Since $p_{H}$ is rather high, this is a very slow detector, yielding a final utility of 6.914 . Comparing to the optimal mixed strategy, this represents an increase in utility of .614 , due to the exploitation of $P_{2}$ 's poor play. Of course, this detector is only good as long as $P_{2}$ sticks to 
his mixed strategy. It is uneffective against a change in the proposers' behaviour: if for instance $p_{H}$ suddenly drops below .725, $P_{1}$ 's utility decreases under 6.3, the guaranteed payoff with the minimax strategy. In this aspect, the Bayes criterion implies a more optimistic attitute of $P_{\mathbf{1}}$.

For the second set of medical information $\left(x_{2}, x_{6}, x_{7}\right)$, the optimal detector $\left(p_{S}=.45, p_{F}=.09\right)$ grants a utility of 7.169 if $p_{H}=.85$, an improvement of .649 comparing to the minimax strategy (see fig. 11).

\section{TOWARDS MORE REALISM}

\subsection{Surcharges}

Conceptually, the introduction of the possibility of accepting a proposer with a surcharge presents little difficulty: it amounts to introduce one more pure strategy for the insurer.

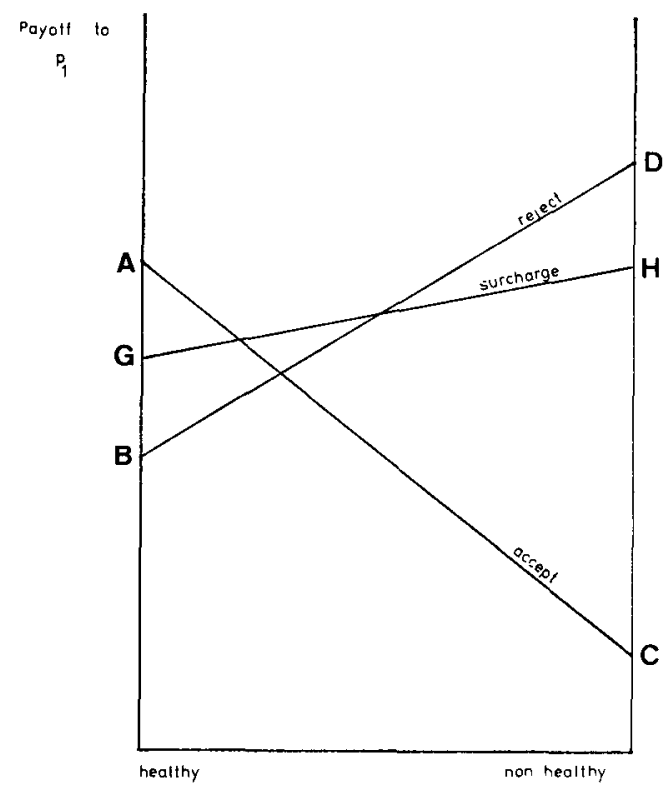

Fig. 13

A detector could then be defined by two critical values $C_{1}$ and $C_{2}$ enveloping an incertitude or surcharge zone.

The two critical limits would determine 4 probabilities

$p_{1}=$ probability of accepting a bad risk

$p_{2}=$ probability of surcharging a bad risk

$p_{3}=$ probability of rejecting a good risk

$p_{4}=$ probability of surcharging a good risk 


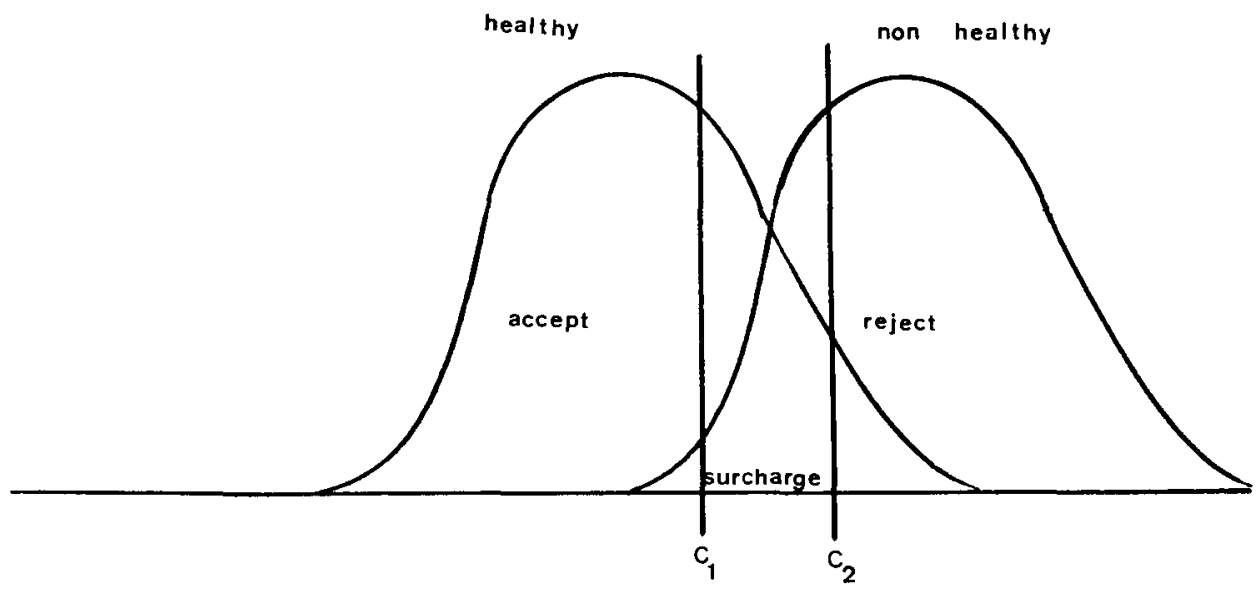

Fig. 14

and two efficiency curves. A necessary condition for a detector to be optimal is that the corresponding payoff line is horizontal, i.e. that

$$
\left(1-p_{3}-p_{4}\right) A+p_{4} G+p_{3} B=\left(1-p_{1}-p_{2}\right) D+P_{2} H+P_{1} C .
$$

The two efficiency curves and (2) determine 3 relations between the probabilities. One more degree of freedom is thus available to maximize the payoff.

\subsection{Increasing the Number of Strategies of $P_{2}$}

In order to practically implement the preceding theory one should subdivide $P_{2}$ 's strategy "present a non healthy proposer" according to the various classes of diseases. $P_{1}$ should then have as pure strategies: reject, accept, a set of surcharges, and follow detector's advice, and $P_{2}$ as many pure strategies as the number of health classes. The graphical interpretation of the game is lost, but linear programming can be used in order to determine its value and optimal strategies.

\section{Appendix: The Likelihood Ratio Method}

Let

- $x$ be the value of the discriminant variable,

$-p(H)$ and $p(N H)$ the a priori probabilities of being healthy or non healthy,

- $f(x \mid H)$ and $f(x \mid N H)$ the conditional distributions of $x$.

We can then compute the a posteriori probability of being non healthy, given the value of the discriminant variable

$$
p=p(N H \mid x)=\frac{f(x \mid N H) p(N H)}{f(x \mid N H) p(N H)+f(x \mid H) p(H)} .
$$


Similarly $p(H \mid x)=1-p$.

The expected payoffs for the two decisions are

$$
\begin{aligned}
& E P_{A}=(1-p) A+p C \\
& E P_{R}=(1-p) B+p D .
\end{aligned}
$$

Define $D^{*}$ to be

$$
D^{*}=E P_{A}-E P_{R}=[(A-B)+(D-C)] p-(A-B) .
$$

Consequently, $D^{*}$ is a linear function of $p$, with a positive slope. There exists a critical $p, p_{c}$, for which $D^{*}=0$ :

$$
p_{c}=\frac{(A-B)}{(A-B)+(D-C)}
$$

and the optimal decision rule is to

- reject if $p>p_{c}$ (then $D^{*}>0$ ) and to

- accept if $p<p_{c}$ (then $D^{*}<0$ ).

If $f(x \mid H)$ and $f(x \mid N H)$ are normal densities with equal variances, there is a one-to-one monotonic relationship between $p$ and $x$, and thus the critical probability $p_{c}$ induces a critical value $x_{c}$.

In general, however, the cutoff point is not unique. There may be two or more critical values.

In that case, we define the likelihood ratio of $x$ for hypothesis $N H$ over hypothesis $H$ as

$$
L(x)=\frac{f(x \mid N H)}{f(x \mid H)}
$$

Of course $0 \leqq L(x) \leqq \infty$.

Substituting $L(x)$ in (1) gives

or

$$
p=\frac{1}{\frac{1}{L(x)} \frac{p(H)}{p(N H)}+1}
$$

$$
L(x)=\frac{p(H)}{p(N H)} \frac{p}{1-p} .
$$

For constant a priori probabilities, there is a monotone relationship between $p$ and $L(x) ; L(x)$ goes from o to $\infty$ as $p$ goes from $o$ to 1 . Therefore, a unique critical likelihood ratio $L_{c}(x)$ exists and can be obtained by replacing $p_{c}$ for $p$ in $(2)$

$$
L_{c}(x)=\frac{p(H)}{p(N H)} \frac{A-B}{D-C} .
$$



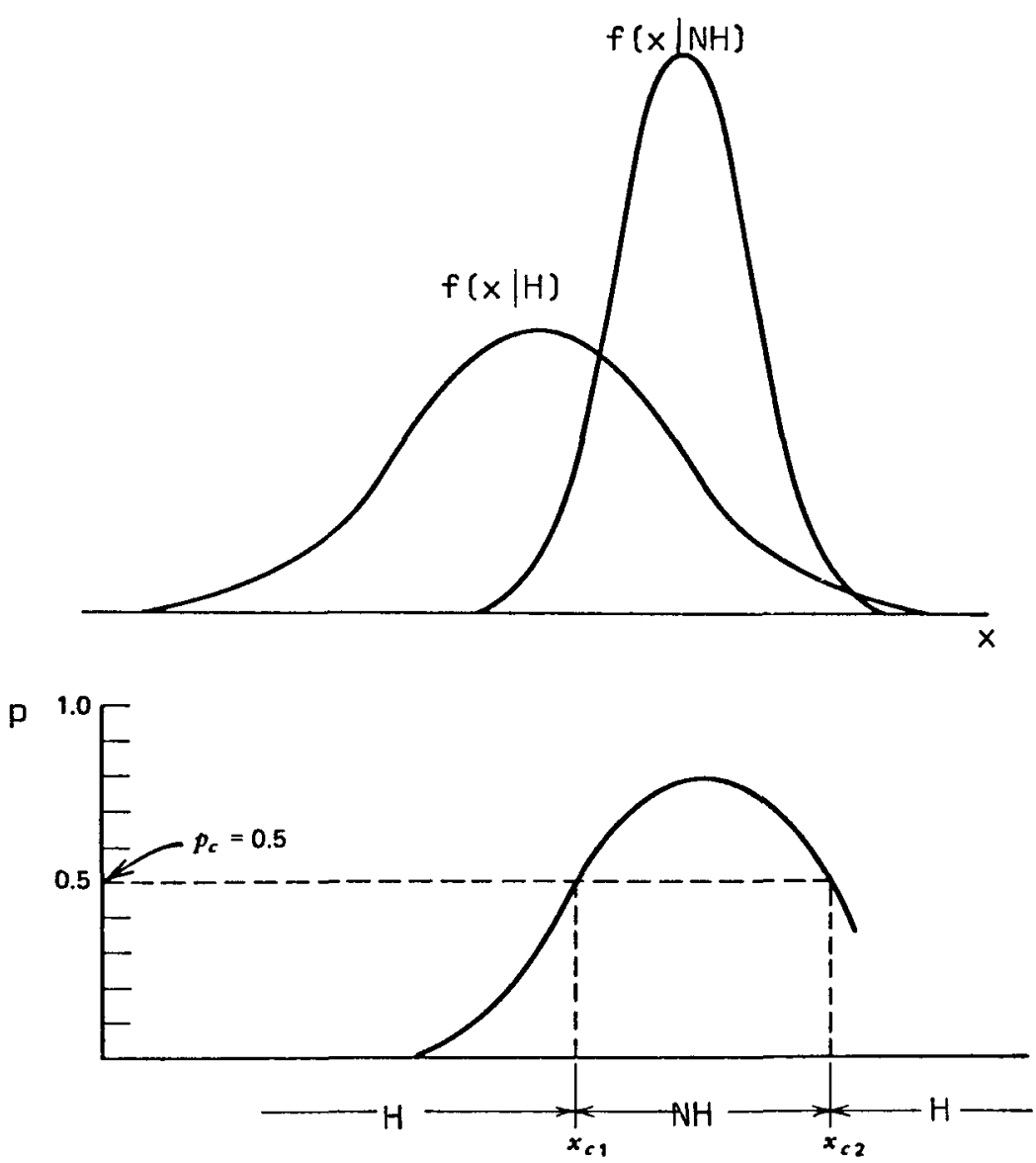

Fig. 15

The optimal decision rule reads

$$
\text { if } L(x)>L_{c}(x) \text {, reject; }
$$$$
\text { if } L(x)<L_{c}(x) \text {, accept. }
$$

Notice that, if $A-B=D-C, p_{c}=1 / 2$. The decision rule is equivalent to maximizing the expected number of correct classifications. From (3)

$$
L_{c}(x)=\frac{p(H)}{p(N H)} .
$$

If, furthermore, the prior probabilities are equal, $L_{c}(x)=1$.

\section{REFERENCES}

Axelrod, R. (1978). Coping with deception, International conference on applied game theory, Vienna.

LEE, W. (1971). Decision theory and human behaviour, J. Wiley, New York.

Luce, R. and H. RAIfFA. (1957). Games and decisions, J. Wiley, New York.

Owen, G. (1968). Game theory, W. Saunders, Philadelphia. 